\title{
The pre-menstrual syndrome
}

\author{
A study in people with a mental handicap
}
R. V. Browne, Consultant Psychiatrist, Coed Du Hospital, Rhydymwyn, near Mold, Clwyd and Bryn y Neuadd Hospital, Llanfairfechan, Gwynedd LL33 0HH; N. C. ELLIS, Lecturer, University College of North Wales, Bangor; and J. A. HIRD, Probationer Clinical Psychologist, Subdepartment of the Department of Clinical Psychology, University of Liverpool

Since its clinical delineation, the premenstrual syndrome has enjoyed a wide repertoire of descriptive labels commensurate with the enormity of its symptomatology and the diversity of its postulated aetiologies, none of which has attained pre-eminence, although some (e.g. progesterone deficiency) have been of more enduring interest, due perhaps as much to the enthusiasm of their authors as to the presence of any compelling scientific premise. The entity thus remains of syndromal status constituting as it does an enigmatic curiosity for some and a fellowship of controversy for others, with little agreement as to its exact nature, time of occurrence or even its existence.

While it is regarded by some as a major clinical entity affecting a large section of the female population, there is nonetheless considerable variation in the reported prevalence of the syndrome, with studies showing as few as $20 \%$ "normal UK women" or as many as $95 \%$ in "normal US women" (Clare, 1982). To date only one clinical survey has included mentally handicapped women (Takayama, 1972). This study used questionnaires and interviews involving some 3,233 Japanese women aged from 14 40 years, 30 of whom were mentally handicapped, and concluded that the syndrome occurred in $77.9 \%$ of the sample, and contrary to traditional understanding in only $8.6 \%$ of cases was the syndrome confined to the premenstruum $(69.3 \%$ continuing to have symptoms after menstruation commenced).

It further concluded that the syndrome (including neuropsychiatric symptoms) occurred as frequently in those with a mental handicap as it did in the group as a whole. As that portion of the survey carried out on those with a mental handicap was conducted by interview (thus necessitating a reasonable level of communicative skill), it is likely that they were on average less handicapped than the population contained within this study. This latter controlled study, conducted exclusively in the mentally handicapped, suggests that social and cultural factors are of themselves insufficient explanation of the syndrome; thus favouring, at least in part, an organic aetiology. This study does, however, have other clinical implications for the assessment and care of people with a mental handicap who also have premenstrual syndrome, which are discussed below.

\section{The study}

Forty-one mentally handicapped residents of Bryn y Neuadd Hospital were observed twice daily by the nursing staff for 103 days. There were 33 women and eight men. On completion of the study, 16 of the females had at some point been observed to menstruate. These individuals constitute the subjects of this report.

The median age was 37 . Eleven were classified severe mental retardate, one autistic and the remainder mild mental retardate. Four of the individuals additionally showed symptoms of schizophrenia. There was a variety of prescribed long-term medication: two pill, two lithium, nine tranquilliser, three anticonvulsant, five antiparkinsonian, one diuretic, one steriod. The communication and expressive skills of these individuals were typically poor and we would not expect them to know or understand about the pre-menstrual syndrome.

The ward staff on each villa were trained to use an observational procedure which was based on the Steiner, Haskett \& Carroll (1980) behavioural checklist. This covers nine subscales; irritability-hostility, tension, dysphoria/depression, aggression, motor co-ordination, efficiency, social impairment, sexual drive and activity, and eating habits. Each of these is rated on a four point (0-3) scale, although scores could be positive or negative for sexual drive and eating habits depending on increasing or decreasing levels of activity. The observers also recorded details of epileptic fits, illnesses, trips out, visits from relatives, menstruation, medication changes, bizarre behaviour and the number of qualified and unqualified staff. The staff were told that the ratings would allow a comprehensive record of behaviour over a three month period and the menstruation question 
was low key and well hidden among all the others. They were trained to use the scale over two weeks. Percentage agreement between two independent observers on these scales ranged from $81 \%$ to $99 \%$.

\section{Findings}

We analysed the rating of these individuals on the nine subscales and an index score (the sum of the subscales) of the Steiner behavioural rating scale over 106 days using SPSS-X. Details of the procedure and findings and their implications for the physiological and culture determinancy of PMS are available from the authors. Because the length of the premenstrual period has been set at widely differing limits by different investigators, we kept open all options by trying three definitions: two day, seven day and 14 day premenstrual phase. We then tested whether levels of activity differed between the premenstrual phase and the rest of the cycle (including menstrual days) using two factor ANOVAs (two-phase ((premenstrual vs. the rest)) $\times 16$ subjects with unequal numbers of replicates within each phase/cell). The analysis for the two longer definitions of premenstrual phase (7 and 14 days) demonstrated no differences in levels of activity between the premenstrual phase and the rest of the cycle. This was true for the Overall Index and for the subscales.

However, when the premenstrual phase was defined as the two days preceding menstruation, there were significant effects. The premenstrual levels on the total Index were significantly higher than in the rest of the cycle [pre-M mean (sd) 1.34 (2.21), mean levels for the rest of the cycle 1.11 (1.93); $P=0.04$ ]. This overall effect is not replicated across all of the nine subscales tension [Pre-M 0.29 (0.60), rest $0.21(0.50) ; P=0.01]$ and dysphoria/depression [pre-M $0.16(0.42)$, rest $0.09(0.31) ; P=$ 0.01]. Significant second order subject by phase interactions $(P=0.05)$ for both tension and dysphoria scales demonstrate that some subjects show more of a premenstrual effect than others, and when we looked for significant within-subject premenstrual effects using fairly conservative MannWhitney $U$ tests it appeared that there were two subjects who showed significant premenstrual increases in overall Index, three in tension and three in dysphoria. These effects did not seem to be associated with either the communicative competence of the individual or her degree of mental handicap: Ss 36 and 4 had complex expressive and comprehension abilities and were both classified as schizophrenic, Ss 3 and 5 only simple comprehension abilities and could at best express themselves using body language. All four individuals were described as being mentally retarded.

\section{Comment}

\section{(a) The association with psychiatric illness} (schizophrenia)

While it is the case that psychiatric patients report significantly more severe distress on the Moos Menstrual Distress Questionnaire than do normal women, (Zola et al, 1979) the positive correlation appears for the most part to be with neuroticism. Indeed, while some (Coppen, 1965) differ in their findings as to the existence of a positive correlation between dysmenorrhea and neuroticism, there is no disagreement as to a positive correlation between premenstrual syndrome and neuroticism. This association is perhaps not surprising in view of the similarity of the symptomatology which tends to be ubiquitous and non-specific in nature and indeed may be common as constitutional traits. The relationship between PMS and psychiatric illness is, however, a one-way phenomenon and hence one is more likely to encounter PMS symptomatology in psychiatrically ill women than to find psychiatric illness in the former.

In this study, two of the four subjects in whom behavioural variance with a cycle phase effect was observed were previously diagnosed as suffering from schizophrenia. (These clinical diagnoses also satisfy Feighner's criteria). In these subjects the upturn in behavioural variance was on the subscale of tension and one of the subjects also showed an increase in the overall index.

In explanation of the association with schizophrenia in this study, two possibilities should be entertained: firstly that the finding is coincidental, or secondly that it may represent a cyclical change in the 'schizophrenic state'. In favour of the former argument, schizophrenic patients are found to have a lower level of dysmenorrhea and premenstrual syndrome than do controls (Coppen, 1965). The relationship of psychiatric illness to PMS is a 'one-way' phenomenon and there is in any event an increased incidence of schizophrenia in a mentally handicapped population.

However, while it may sometimes be the case that affective disorders are exacerbated premenstrually, it may not be unreasonable to consider the upturn in tension as indicative of a cyclical deterioration in the schizophrenia illness. In support of this latter possibility, patients with schizophrenia (especially those with a chronic illness) are on average more aroused than normal subjects and also show an abnormality in the way their attention and arousal co-vary (Claridge, 1972) and hence the possibility that schizophrenic symptoms as a whole co-vary with the menstrual cycle. This cyclical increase in arousal could thus be incorrectly perceived as an upturn in tension on the part of the observer. 


\section{(b) Quantitative and qualitative aspects}

The rather high incidence in the group surveyed by Takayama (1972) compared with the incidence of $25 \%$ in this study is likely to be due at least in part to definition, as the majority of the subjects in that survey experienced symptoms even after the onset of menstruation and hence those findings could be more representative of a paramenstrual syndrome.

As the median age of 37 years in our study is in keeping with the tendency for complaints to be more frequently made by women in their thirties and forties one may expect a high incidence. However, there are several reasons why the incidence in this study may constitute an underestimate. Not only is the interpretation of symptoms and the diagnosis of mental illness in the mentally handicapped a more difficult and arduous process, but the necessity for observer rating, the smaller repertoire of available symptomatology upon which to base a diagnosis, the reliability achieved, and the fact that some symptoms may be suppressed by the use of psychotropic medication (thus providing a more occult clinical picture) are additional factors.

Accepting that PMS be a syndrome of limited clinical expression in the mentally handicapped, it does seem that the main difference may nonetheless be of a quantitative nature, at least inasmuch as tension and dysphoria are among those psychological symptoms most frequently encountered in PMS in the general population (Steiner et al, 1980). Those other symptoms of PMS present in the normal population are either not among the repertoire of symptoms experienced by people with a mental handicap and suffering from PMS or are inaccessible to the clinician for a variety of reasons, not least because of linguistic/communicative difficulties which contribute to the difficulty of recognising and interpreting symptoms in those with a mental handicap. Indeed, an adaptation of the 'general model of anxiety (Lader, 1975) could perhaps go a long way in providing and understanding of PMS in this group where both environmental and internal factors may be of aetiological significance.

\section{Concluding remarks}

Given the occult nature of all types of illness occurring in the mentally handicapped due to communicative difficulties and the limited clinical expression of
PMS in people with mental handicap, a "positive investigative stance" is required (none of the subjects in this study was previously diagnosed as suffering from PMS). Of course, no laboratory test exists and there are no pathonomonic features but increased awareness and the use of a diagnostic format may be helpful.

As periodicity/cycle phase affect is a prerequisite to diagnosis, the use of a menstrual diary is required. While this of necessity must be kept by the nurse/care giver (and is therefore a record of observed behaviour), it should have regard to all observable symptomatology commensurate with PMS in the general population, together with a recognition of tension and dysphoria as possible diagnostic indicators. Given the limitations associated with the diagnostic process in this group, the use of too strict operation criteria (as that of Steiner) may not be appropriate if a humane approach is to be maintained.

\section{Acknowledgements}

We are indebted to those nursing staff at Bryn y Neuadd Hospital who were involved in the collection of the data.

\section{References}

Clarke, A. W. (1982) Premenstrual tension. In Handbook of Psychiatry, volume 2, chapter VIII (ed. G. F. M. Russell). Cambridge University Press.

ClaRIDGE, G. (1972) The schizophrenics as nervous types. British Journal of Psychiatry, 121, 1-17.

COPPEN, A. (1965) The prevalence of menstrual disorders in psychiatric patients. British Journal of Psychiatry, 111, 155-167.

LADER, M. H. (1975) Psychophysiological aspects of anxiety. Medicine (second series), no. 10, 429-432.

Steiner, M., Haskett, F. G. \& Carroll, B. J. (1980) Premenstrual tension syndrome: the development research diagnostic criteria and new rating scales. Acta Psychiatrica Scandinavica, 62, 177-190.

TAKAYAMA, T. (1972) A clinical study of premenstural syndrome. In Psychosomatic Medicine in Obstetrics and Gynaecology (ed. N. Morris). Basel: Karger.

Zola, P., Meyerson, A. T., ReZnikoff, M. et al (1979) Menstrual symptomatology and psychiatric admission. Journal of Psychosomatic Research, 23, 241-245.

A full list of references is available on request to the senior author. 